\title{
Renal primitive neuroectodermal tumour: Case series and brief review
}

\author{
Tadeusz Kroczak, MD; Rajan Sharda, MD;† Darrel Drachenberg, MD, FRCSC;* Turki Al-Essawi, MD
}

*Section of Urology, Department of Surgery, St. Boniface Hospital University of Manitoba, Winnipeg, MB; ‘'Section of Urology, Department of Surgery, Cornwall Community Hospital, Cornwall, ON; §Section of Urology, Department of Surgery, Health Sciences Centre University of Manitoba, Winnipeg, MB

Cite as: Can Urol Assoc J 2014;8(3-4):e241-4. http://dx.doi.org/10.5489/cuaj.1770 Published online April 14, 2014.

\section{Abstract}

Renal primitive neuroectodermal tumor is a rare malignancy. These tumours rarely present with caval involvement. We report 2 cases of primitive neuroectodermal tumours (PNETs) with inferior vena cava involvement. The initial presentation and outcomes differed significantly. The diagnosis was confirmed using histologic and pathologic analysis. We present a brief literature review and an outline of typical clinical and pathologic features of renal PNETs.

\section{Case 1}

A 17-year-old previously healthy male presented to the emergency department at the Health Sciences Centre with a 2-month history of persistent cough, 10-lb weight loss and 1 month of abdominal bloating. The patient also described an episode of gross hematuria after exercising. Physical exam revealed a non tender abdomen with fullness in the left upper quadrant. Initial blood work showed a creatinine (Cr 93) and mild anemia (Hgb 98). The patient's international normalization rate (INR) (1.8) and lactic acid dehydrogenase (LDH) (530) were elevated; however, all other liver function tests were normal.

A computerized tomography (CT) chest and abdomen were performed for further workup. We noted a large left renal mass measuring $14 \times 11 \mathrm{~cm}$ with tumour thrombus extending to the infrahepatic inferior vena cava (IVC) with multiple regional metastatic lymph nodes, as well as lung metastasis (Fig. 1). A bone scan revealed no osseous metastatic involvement.

The patient underwent left radical nephrectomy, IVC tumour thrombectomy and retroperitoneal lymphadenectomy. The pathology revealed a $14.8 \times 14 \times 12$-cm tumour involving the entire left kidney, contained within Gerota's fascia and negative surgical margins. Two out of 11 lymph nodes were positive for malignancy. Initial immunochemistry showed tumour cells positive for vimentin, CD99, CD56 and p53 and negative for CD45, WT1, desmin and MyoD1. This suggested a poorly differentiated sarcoma or Ewing's family tumour. Molecular test by reverse transcriptase was performed and was positive for EWSR1-FLI1, which confirmed the diagnosis of Ewing's sarcoma/primitive neuroectodermal tumour (PNET).

The patient is now 12 months post-surgery and has received 12 cycles of chemotherapy on an alternating regimen of cyclophosphamide, adriamycin and vincristine (CAV) interchanged with ifosphamide/etoposide every 2 weeks. The patient has had a dramatic response to chemotherapy with regression of pulmonary metastases. Unfortunately, the patient has developed ifosfamide-induced Fanconi syndrome and nephrotoxicity. His serum creatinine is 630 and he may require dialysis.

\section{Case 2}

A 31-year-old male with a medical history of hypertension, recent diagnosis of deep vein thrombosis of the leg and reactive airway disease presented to a walk-in clinic with several weeks of general malaise, flank pain and gross hematuria. The patient had also experienced leg pain and cramping along with shortness of breath. An abdominal ultrasound revealed a renal mass and a Doppler ultrasound of the right lower extremity confirmed a deep venous thrombus involving the right lower extremity with nearly completely occlusive thrombus.

Physical examination revealed a palpable mass in the right upper quadrant with no associated tenderness. No palpable lymphadenopathy or peripheral edema was found. Bloodwork revealed a normocytic anemia (Hgb 111) and an elevated serum creatinine ( $\mathrm{Cr} 127)$. Alkaline phospha- 


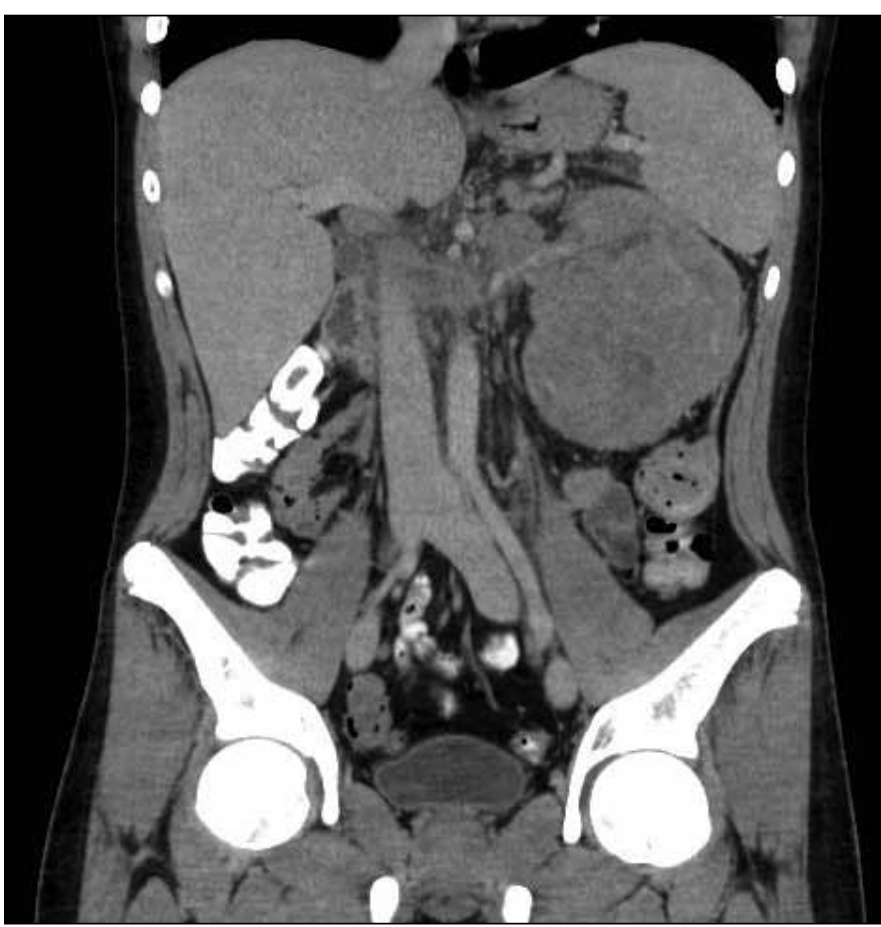

Fig. 1a. Computed tomography axial view showing left renal mass with extension of tumour into renal vein and vena cava.

tase (ALP 178), Gamma-glutamyl transpeptidase (GGT 492), and LDH (384) were all elevated. INR was elevated at 1.6. Several imaging studies were ordered to evaluate the extent of metastatic disease and for surgical planning. A CT abdomen demonstrated a $17 \times 13-\mathrm{cm}$ right renal mass with tumour thrombus extending to the intrahepatic cava and inferiorly to both common illiacs. Subsequent magnetic resonant imaging (MRI) of the abdomen and pelvis showed the renal mass with the tumour extending up the IVC and contralaterally into the left renal vein orifice. Extensive bland tumour thrombus was present within the cava and iliac veins below the tumour. No hepatic metastases were seen. The chest $\mathrm{x}$-ray was unremarkable, however a CT thorax revealed small clusters of branching nodular opacities in the right lung. No evidence of pulmonary embolus was seen. Bone scan was negative for skeletal metastases.

The patient was admitted to hospital for urgent surgery and anticoagulation. An open right radical nephrectomy, caval thrombectomy, resection of the vena cava and retroperitoneal lymph node dissection were preformed. The IVC was tied off at its bifurcation and the renal and suprarenal cava resected en block with the mass. Bland thrombus was removed from the proximal cava prior to ligation. Intraoperative inspection of the gallbladder revealed acute on chronic inflammation with cholelithiasis. A cholecystectomy was performed as well. The patient tolerated the surgery quite well after an estimated blood loss of $5 \mathrm{~L}$ requiring hematologic support.

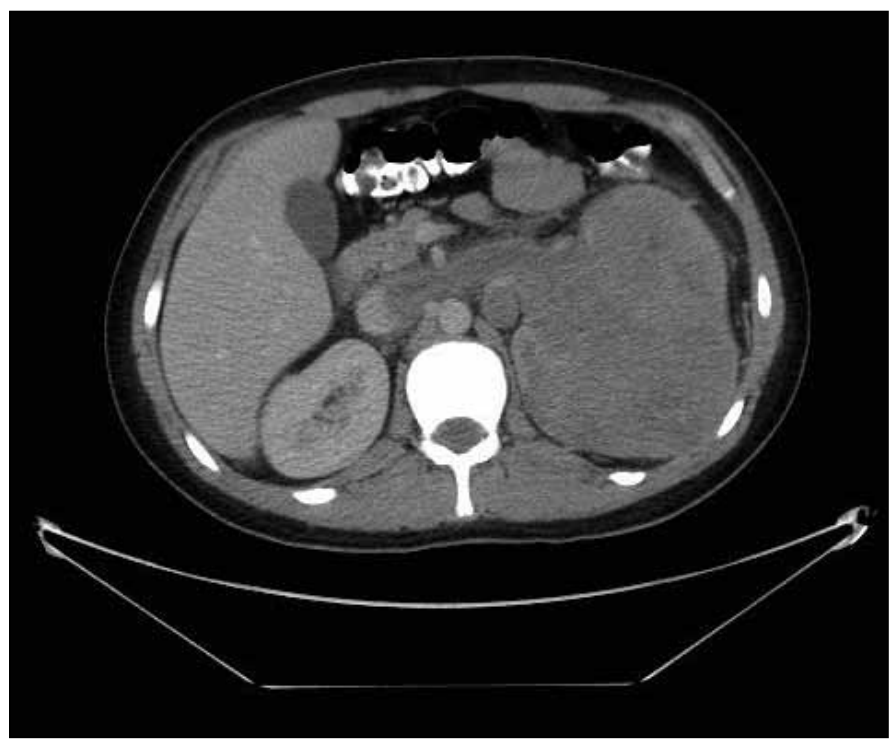

Fig. $1 \boldsymbol{b}$. Computed tomography coronal view showing left renal mass with extension of tumour into renal vein and vena cava.

Pathology revealed a $17-\mathrm{cm}$ tumour involving the right kidney with renal capsule and peri-renal fat involvement along with renal vein and vena cava invasion. There were no positive lymph nodes and no involvement of Gerota's fascia. Tumour cells were strongly positive for vimentin, focal positivity for CD99, BCL-2, NSE, focal weakly positive for Synaptophysin, CD 56, S-100, but negative for WT1, actin, chromogranin, CK7, CK20, p53, CD10, EMA, inhibin, and CD 45. Molecular tests were performed and showed positivity for EWSR1-FLI1 by reverse transcriptase protein:creatinine ratio. These findings confirmed the diagnosis of Ewing's sarcoma/PNET.

The patient was referred to medical oncology and adjuvant chemotherapy of 6 cycles of CAV (cyclophosphamide, adriamycin, and vincristine), alternating with 6 cycles of VP-16 ifosamide. Consultation with radiation oncology was also obtained; however radiation was not offered. Imaging after the initiation of chemotherapy showed pulmonary, hepatic, and abdominal lymphadenopathy consistent with metastases. After completion of this chemotherapeutic regimen, the patient had unfortunately continued disease progression and was switched to gemcitabine/docetaxel. Palliative care started 1 year after surgery and the patient died of the disease shortly thereafter.

\section{Discussion}

PNETs are small round cell tumours that arise from cells of the primitive ectoderm and comprise $1 \%$ of all sarcomas. These tumours are part of the Ewing's sarcoma/PNET family of tumours and exhibit neuroepithelial differentiation. Their location is typically in the trunk or axial skeleton as a bone or soft tissue mass, and rarely in the genitourinary system. 
The first report of renal PNET was by Seemayer and colleagues and to date there have been few reports. ${ }^{1,2-7}$ There are even fewer cases of renal PNET with extension into the IVC. ${ }^{8-11}$ PNETs are characterized by an aggressive clinical course and poor prognosis. The mean patient survival is about 10 months. ${ }^{12}$ The first patient we presented not only had IVC extension, but also showed remarkable response to treatment. This patient has survived 12 months to date and has had a dramatic response to treatment. The second patient also had renal vein involvement; however invasion into the vena cava was also noted along with bland tumour thrombus extending bilaterally to the iliac veins. To the best of our knowledge, there have only been 2 other case reports of caval thrombectomy in patients with PNET. ${ }^{13}$

Renal PNETs typically occur in young adults. Patients with renal PNET present with non-specific symptoms which may include malaise, fever, flank pain, hematuria, night sweats, and dyspnea. Serum biochemistry values may show elevated LDH, glutamicoxaloacetic transaminase, glutamicpyruvic transaminase, and creatinine. ${ }^{13}$ These tumours have various different macroscopic presentations. Gross inspection reveals a lobular shape with either dark brown, yellow, or gray-white areas of necrosis or hemorrhage. ${ }^{13}$ The histologic hallmark of PNET is formation of Homer-Wright rosettes or pseudorosettes involving hyperchromatic cells. Several immune markers may be detected which include neuron-specific enolase (NSE), vimentin, synaptophysin and S-100. The immune marker CD-99 is present in virtually all tumours. ${ }^{13}$ The most common genetic mutation in PNETs is $\mathrm{t}(11 ; 22)(\mathrm{q} 24 ; \mathrm{q} 12)$ and the second most common being $\mathrm{t}(21 ; 22)(\mathrm{q} 22 ; \mathrm{q} 12) .{ }^{14}$ Molecular testing is helpful in situations with a confusing immunohistochemical profile. The diagnosis of renal PNET must include tumour morphology, immunostaining profile and occasionally genetic mutations.

Treatment may be a combination of surgery, chemotherapy and radiotherapy. ${ }^{12,13}$ Renal PNET is treated with similar chemotherapeutic agents used with Ewing's sarcoma given their biologic similarities. The typical chemotherapeutic agents used are vincristine, dactinomycin, adriamycin, cyclophosphamide, ifosamide and etoposide. ${ }^{15}$ The addition of ifosfamide and etoposide has been shown to improve overall survival in patients with non-metastatic skeletal Ewing's sarcoma. ${ }^{16}$ Neoadjuvant chemotherapy can improve surgical outcomes and potentially avoid adjuvant radiotherapy, which is often reserved for patients with positive margins after resection or involvement of Gerota's fascia. ${ }^{17,18}$

\section{Conclusion}

These 2 case reports are unique as there have only been a few published case reports of renal PNETs with caval involvement. Both patients had varying clinical presenta- tions and outcomes. Renal PNETs are rare tumours with a poor prognosis. These cases outline the difficulty in diagnosis, management, and variation in natural history. Unique histologic features, immunostaining profile, and genetic features are important in making the histologic diagnosis. Our report shows that a multidisciplinary approach is essential in the management of renal PNET, and some patients may respond more favourably than previously thought.

Competing interests: Dr. Kroczak, Dr. Sharda and Dr. Al-Essawi all declare no competing financial or personal interests. Dr. Drachenberg has attended Advisory Boards for Astellas and Janssen and has been a speaker for Amgen and Actavis (formerly Watson). He has also been an investigator in clinical trials run by Cancer Care Manitoba (CCMB).

This paper has been peer-reviewed.

\section{References}

1. Seemayer TA, Thelmo WL, Bolande RP. Peripheral neuroectodermal tumors. Perspect Pediatr Pathol 1975;12:151-2.

2. Parham DM, Roloson $\mathrm{GJ}$, Feely $M$, et al. Primary malignant neuroepithelial tumors of the kidney: A clinicopathologic analysis of 146 adult and pediatric cases from the National Wilms' tumor Study Group Pathology Center. Am J Surg Pathol 2001;25:133-46. http://dx.doi.org/10.1097/00000478200102000-00001

3. Dogra PN, Goel A, Kumar R, et al. Extraosseous Ewing's sarcoma of the kidney. Urologia Int 2002;69:1502. http://dx.doi.org/10.1159/000065566

4. Saxena R, Sait S, Mhawech-Fauceglia P. Ewing sarcoma/primitive neuroectodermal tumor of the kidney: A case report. Diagnosed by immunochemistry and molecular analysis. Ann Diagn Pathol 2006;10:363-6. http://dx.doi.org/10.1016/i.anndiagpath.2005.11.001

5. Jimenez RE, Folpe AL, Lapham RL, et al. Primary Ewing's sarcoma/primitive neuroectodermal tumor of the kidney: A clinicopathologic and immunohistochemical analysis of 11 cases. Am I Surg Pathol 2002;26:320-7. http://dx.doi.org/10.1097/00000478-200203000-00005

6. Kang SH, Perle MA, Nonaka D, et al. Primary Ewing sarcoma/PNET of the kidney: Fine needle aspiration, histology, and dual color break apart FISH assay. Diag Cytopathol 2007;35:353-7. http://dx.doi. org/10.1002/dc.20642

7. Bing Z, Zhang P, Tomaszewski JE, et al. Primary Ewing sarcoma/primitive neuroectodermal tumor of the kidney. J Urol 2009;181:1341-2. http://dx.doi.org/10.1016/i.uuro.2008.12.029

8. Fergany AF, Dhar N, Budd GT, et al. Primary extraosseous Ewing sarcoma of the kidney with level iii inferior vena cava thrombus. Clin Genitourinary Cancer 2009;7:95-7. http://dx.doi.org/10.3816/ CGC.2009.n.032

9. Castro EC, Parwani AV. Ewing sarcoma/primitive neuroectodermal tumor of the kidney: Two unusual presentations of a rare tumor. Case Rep Med 2012:1-7. http://dx.doi.org/10.1155/2012/190581

10. Thomas IC, Sebek BA, Krishnamurthy V. Primitive neuroectodermal tumor of the kidney with inferior vena cava and atrial tumor thrombus. J Urol 2002;168:1486-7. http://dx.doi.org/10.1016/S0022$5347(05) 64487-3$

11. Karnes RJ, Gettman MT, Anderson PM, et al. Primitive neuroectodermal tumor (extraskeletal Ewing's sarcoma) of the kidney with vena caval tumor thrombus. J Urol 2000;164:772. http://dx.doi. org/10.1016/S0022-5347(05)67301-6

12. Cuesta Alcala JA, Solchaga Martinez A, Caballero Martinez MC, et al. Primary neuroectodermal tumor (PNET) of the kidney: 26 cases. Current status of its diagnosis and treatment [in Spanish]. Arch Esp Urol 2001;54:1081-93.

13. Ellinger J, Bastian PJ, Hauser $S$, et al. Primitive neuroectodermal tumor: Rare, highly aggressive differential diagnosis in urologic malignancies. Urology 2006;68:257-62. http://dx.doi.org/10.1016/i. urology.2006.02.037

14. de Alava E, Gerald WL. Molecular biology of the Ewing's sarcoma/primitive neuroectodermal tumor family. J Clin Oncol 2000;18:204-13. 
Kroczak et al.

15. Thyavihally $Y B$, Tongaonkor $H B$, Gupta $S$, et al. Primitive neuroectodermal tumor of the kidney: $A$ single institution series of 16 patients. Urology 2008;71:292-6. http://dx.doi.org/10.1016/i.urology.2007.09.051

16. Grier HE, Krailo MD, Tarbell NJ, et al. Addition of ifosfamide and etoposide to standard chemotherapy for Ewing sarcoma and primitive neuroectodermal tumor of the bone. N Engl J Med 2003;348:694-701. http://dx.doi.org/10.1056/NEJMoa020890

17. Sciubba DM, Okuno SH, Dekutoski MB, et al. Ewing and osteogenic sarcoma: Evidence for multidisciplinary management. Spine 2009;34:S58-68. http://dx.doi.org/10.1097/BRS.0b013e3181ba6436
18. Shamberger RC, LaQuaglia MP, Gebhardt MC, et al. Ewing sarcoma/primitive neuroectodermal tumor of the chest wall: impact of initial versus delayed resection on tumor margins, survival, and use of radiation therapy. Ann Surg 2003;238:563-7; discussion 567-8.

Correspondence: Dr. Tadeusz Kroczak, Section of Urology, Department of Surgery, St. Boniface Hospital University of Manitoba, Winnipeg, MB; umkrocza@cc.umanitoba.ca 